\title{
"On the Integration of MPEG-4 Streams Pulled Out of High Performance Mobile Devices and Data Traffic over a Wireless Network"
}

\author{
Spyros Psychis, Polychronis Koutsakis, and Michael Paterakis \\ Electronics \& Computer Engrg. Dept. \& \\ Telecommunication Systems Institute \\ Technical University of Crete \\ 73100, Chania, Greece \\ \{psycho, polk, pateraki\}atsinet.gr
}

\begin{abstract}
In this work we propose and evaluate mechanisms for the multiplexing and the integrated delivery of Video and Data Traffic over a Wireless Cellular High Speed Packet Switched Network. We envision a system where the Mobile Terminals are considered to be high performance devices with extended storage capabilities which can act like cache memories streaming multimedia material. We focus on the uplink channel and we thoroughly investigate the system's performance under a variety of possible loads which consist of actual MPEG-4 streams and Data Traffic.
\end{abstract}

Keywords: Wireless Packet Switched Networks, TDMA, MAC, Media Access Control, MPEG-4 streams

\section{Introduction}

The coexistence of wired and wireless communication networks is a hint that none of the two network types is able to service, on its own, all of the users needs. The basic advantage of wireless communication is that it allows the users to move, even during the time they use the network services. The goal of wireless communication networks is to allow user access to the capabilities of the wired networks at any time without regard to location or mobility. Thus, problems and issues that until now appeared only on wired networks now must be solved on wireless and vice versa. And of course the main concern in wireless communication is the same like in wired: scaling, in other words how to extend the broadband frontier to the end user. Therefore, the system capacity needs to grow as much as the demands of the various services grow.

System capacity can be increased by:

a. using a cellular structure with a cell size as small as possible (microcells) in order to reduce transmitting power and to increase frequency reuse. Microcell diameters are usually of the order of a few hundred meters; therefore the round-trip propagation delay within a microcell is negligible (of the order of $1 \mu$ s or less),

b. using efficient medium access control (MAC) protocols to exploit the variations in access and service required by the disparate user sources. 
Within the microcell, spatially dispersed mobile terminals (MTs) share a radio channel that connects them to a fixed base station or a wireless hotspot. The base station allocates the channel resources (transmission scheduling), delivers feedback information and serves as an interface to the mobile switching center (MSC), which provides access to the fixed network infrastructure and the Internet.

Another important problem is the optimization of the usage of the system capacity.

During the last five years a lot of work has been done on various caching schemes that permit the fair and effective sharing of the network resources [1,2,3]. Most of them have been deployed especially for the retrieval and delivery of high quality stored multimedia content. And since the notion of broadband access is transferred to the wireless field, most of these caching architectures must be extended. In this work, Mobile Terminals are considered to be high performance devices with extended storage capabilities which can act like cache memories, streaming multimedia material to other MT's (in the same or in adjacent micro cells) in order to reduce client start up latencies and improve the network performance. Even the whole micro cell can be faced as a cache farm from the rest of the network. Therefore, we envision high quality stored video streaming on the uplink channel (MT's to the BS) in order for these streams to be delivered for playback to their destinations.

The delivery of high quality stored video material is a service with very strict QoS requirements. Consequently the design of MAC strategies that integrates this class of service with others, which often require contradictory QoS guaranties, is necessary.

As mentioned earlier, in this work we focus on the uplink (mobiles to base station) channel, where a MAC scheme is required in order to resolve the source terminals contention for channel access.

As for the downlink channel, since the base station is the sole transmitter on that channel, it is in complete control of the downstream traffic, using TDMA to relay information to the users.

\section{MPEG-4 and Media Streaming}

MPEG-4 is an ISO standard that provides a standardized and worldwide-accepted technological framework that enables the integration of the production, storage distribution and content access paradigms. MPEG-4 leverages existing digital video content by supporting the MPEG-1 and MPEG-2 coding standards. Furthermore, it enables richer development of new digital video applications and services. An MPEG-4 scene is composed by a number of audiovisual objects, which can be either static or time-varying in nature. [4]

MPEG-4 Visual provides a natural video coding algorithm that is capable of operation from $5 \mathrm{kbps}$ with a spatial resolution of QCIF (144x176 pixels). It is ITU-T H.263 compatible, in the sense that an MPEG-4 video decoder also correctly decodes an H.263 bit stream. The algorithm scales up to bit rates of some Mbit/s and optimization has been carried for ITU-R 601 resolution pictures $(288 \times 720 @ 50 \mathrm{~Hz}$ and 240x720@59.94 Hz). The recently developed Studio Profile can operate at over 1 Gbit/s. In addition MPEG-4 has a so-called Fine Granularity Scalability mode that allows transmission of the same video content at different bit rates from one coded version of the movie [10]. 
Table 1.

\begin{tabular}{|c|c|c|}
\hline Movie Name & $\begin{array}{l}\text { Mean Bit rate } \\
(\text { Kbps })\end{array}$ & $\begin{array}{l}\text { Peak Bit rate } \\
(\text { Kbps })\end{array}$ \\
\hline $\begin{array}{c}\text { News } \\
\text { Cam }\end{array}$ & 720 & 3400 \\
\hline $\begin{array}{c}\text { Silence of } \\
\text { the Lambs }\end{array}$ & 580 & 4400 \\
\hline $\begin{array}{c}\text { The } \\
\text { Simpsons }\end{array}$ & 1300 & 8800 \\
\hline Soccer & 1100 & 3600 \\
\hline Star Wars & 280 & 1900 \\
\hline $\begin{array}{c}\text { Movie Name } \\
(\text { Kbps })\end{array}$ & $\begin{array}{l}\text { Peak Bit rate } \\
(\text { Kbps })\end{array}$ \\
\hline News & 720 & 3400 \\
\hline $\begin{array}{c}\text { Parking Lot } \\
\text { Cam }\end{array}$ & 790 & 2800 \\
\hline $\begin{array}{c}\text { Silence of } \\
\text { the Lambs }\end{array}$ & 580 & 4400 \\
\hline $\begin{array}{c}\text { The } \\
\text { Simpsons }\end{array}$ & 1300 & 8800 \\
\hline Soccer & 1100 & 3600 \\
\hline Star Wars & 280 & 1900 \\
\hline
\end{tabular}

In our study, we use the trace statistics of actual MPEG-4 streams from [11]. The video streams that we used, were carefully selected in order to cover a broad range of movie characteristics. Table 1 shows the movies we used together with their mean and peak bit rates.

Figures 4 through 9 (in page 9) show the histograms of the bit rate of each movie. Horizontal axis shows for each video frame the corresponding bit rate quantized at 1 Kbps sectors. Vertical axis shows the number of video frames that correspond to a specific bit rate. Histograms delineate the bit rate behavior for each movie; a spread distribution corresponds to a bursty stream. Notice that the axes are not necessarily under the same scale.

All the streams are encoded at 25 video frames per second, which means that a new video frame is generated every 40 msecs. Since the video streams are pulled out from Mobile Terminals that act like caches and not dedicated cache engines with optimization or smoothing functionalities, no packet shaping mechanisms were used. Video content is delivered to the network in the initial form that was encoded. In our study we have made the assumption that the maximum transmission delay for video packets is 40 msecs, with packets being dropped when this deadline is reached. That is, all video packets of a VF must be delivered before the next VF arrives. The allowed video packet dropping probability is set to 0.0001 from [5]. 


\section{Data Traffic}

We adopt the data traffic model based on statistics collected on email usage from the Finish University and Research Network (FUNET) [6]. The model simulates email data traffic. The probability distribution function $f(x)$ for the length of the data messages was found to be well approximated by the Cauchy $(0.8,1)$ distribution. The message inter-arrival time distribution for the FUNET model is exponential. We assume an upper limit on the average data message delay equal to 2 seconds.

\section{Frame Structure, BS Scheduling, and Terminal Actions}

The uplink channel time is divided into time frames of equal length. Each frame consists of two types of intervals. These are the request intervals and the information intervals. Each of these intervals is divided into a number of time slots.

Within an information interval, each slot accommodates exactly one, fixed length, packet that contains video or data information and a header. Each request slot is subdivided into two mini-slots and each mini-slot accommodates exactly one, fixed length, request packet. By using more than one mini slot per request slot, a more efficient usage of the available request bandwidth is possible, [7]. The base station broadcasts a short binary feedback packet at the end of each mini-slot indicating only the presence or absence of a collision within the mini-slot (collision (C) versus noncollision (NC)). Upon successfully transmitting a request packet the terminal waits until the end of the corresponding request interval to learn of its reservation slot (or slots). If unsuccessful within the request intervals of the current frame, the terminal attempts again in the request intervals of the next frame. A terminal with a reservation transmits freely within its reserved slot [5].

We adopt the idea that video and data terminals can share the request slots (first by the video terminals, and after the video contention period, by the data terminals). The reason we require video terminals to contend in order to reserve information slots is that we are dealing with high quality stored video content which, in contrast to videoconference streams, generates highly bursty information. Video requirements change frequently over time and it is impossible to ensure that their demands will reach the BS successfully if we use techniques similar to the ones described in [7],[8]. The two-cell stack reservation random access algorithm [9] is used to resolve the collisions, among video request packets.

After the end of the video contention period, data terminals without a reservation and with message ready for transmission, begin their contention in order to reserve information slots. In the case that two or more data request packets collide, the twocell stack random access algorithm [14] is used to resolve the collision, due to its operational simplicity, stability and high throughput.

The BS allocates channel resources at the end of the corresponding request interval, and follows a different allocation policy for video terminals than that for data terminals.

Video terminals have higher priority in acquiring the slots they demand. If a full allocation is possible, the BS then proceeds to allocate any still available information slots to the requesting data terminals. Since we assume here that data slot reservations 
can not be preempted in favor of video terminals waiting for transmission, we choose not to allocate more than one slot per frame to a requesting data terminal. Otherwise, if a full allocation is not possible, the BS grants to the video terminals as many of the slots they requested as possible (i.e., the BS makes a partial allocation). The BS allocates the earliest available information slots to the video terminals, which, if needed, keep these slots in the following channel frames, until the next video frame (VF) arrives.

There are two reasons for adopting the partial allocation policy, described above, to the video users. The first is that in our system there are 10 available reservation slots (20 minislots) in each channel frame, which practically means that almost all of the possible collisions among video terminal request packets can be resolved within the contention period of one channel frame. The second reason is that the video terminals are transmitting high quality bursty video. The size of a video frame is usually of the order of a few kilobytes, which means that after segmentation it corresponds to a few hundred channel cells (of ATM size). Especially in MPEG-4 coding, the correlation factor between two adjacent video frames, is extremely high. Therefore, even in the case that a few tens of cells do not reach the destination; the decoder can successfully reconstruct the video frame. Additionally, MPEG-4 incorporates very capable error correction techniques and can therefore suffer relatively higher packet loss rates compared to other media standards without serious degradation of the playback quality. Consequently, we try to conform to the QoS requirement on packet dropping while spreading the packet losses in time. This spreading of losses can lead to an imperceptible blurring during video playback, which is expected to be acceptable when watching a movie, instead of concentrating the lost cells in time, and consequently receiving video "hiccups" and severe losses in the decoder.

When a video terminal wants to decrease its bit rate, it releases all the slots that were previously allocated to it and are no longer necessary. The BS realizes the change in the bit rate when the first currently unnecessary reserved slot is released by the video terminal (and is observed empty by the BS in the current frame), and consequently allocates the newly released slots to any other requesting terminals, after the end of the request interval of the next frame

When the bit rate of a video terminal increases then the terminal must enter the contention process in order to acquire the additional information slots it needs. In such case, the terminal releases all of its currently reserved information slots before entering the contention period. When it passes the contention period successfully, then the required information slots are reserved and the video terminal can freely transmit within them. The rationale behind this policy is fairness. We prefer to let each video terminal compete with other video terminals at an equal basis (since video has absolute priority), in order to "spread" the video packet losses uniformly.

\section{System Parameters}

The channel rate is $20 \mathrm{Mbps}$ (from [12]). As mentioned before, in this study we investigate the case where the system traffic includes video and data. Nevertheless, high capacity wireless channels will often be used for integrating voice, video and data traffic, and the frame duration is usually chosen to be equal to the time a voice terminal needs to generate a new voice packet [13]. 
Table 2.

\begin{tabular}{|c|c|c|c|}
\hline \multicolumn{2}{|c|}{ Movie } & $\lambda$ & Throughput \% \\
\hline A & News & 5.1 & 86.47266 \\
\hline B & Parking Lot Cam & 4.85 & 83.91547 \\
\hline C & $\begin{array}{c}\text { Silence of the } \\
\text { Lambs }\end{array}$ & 4.78 & 79.1234 \\
\hline D & The Simpsons & 3.1 & 68.67518 \\
\hline E & Soccer & 5.05 & 92.85171 \\
\hline F & Star Wars & 5.85 & 88.76075 \\
\hline & Average & 4.788333 & 83.29986 \\
\hline
\end{tabular}

Assuming a speech codec rate of $32 \mathrm{Kbps}$ and that the information packet length is equal to the size of an ATM cell, yields the channel frame duration of $12 \mathrm{~ms}$. The 12 $\mathrm{ms}$ of frame duration accommodate 566 slots (556 information slots plus 10 reservation slots). Consequently, the above channel's information payload transmission speed is slightly above $18 \mathrm{Mbps}$ (because of the ATM headers encapsulation and the existence of reservation slots).

Finally, given that the packet length is equal to the size of an ATM cell, it can be seen that the average data message length is equal to 80 packets.

\section{Simulation Results and Discussion}

An extensive simulation study was carried out. Each run simulated one hour of actual network activity (300005 channel frames). We simulated the system under all possible movie loads from 1 to 6 movies. For each load, all different combinations were examined (63 in total), from 5 times each (Monte Carlo method). The lack of similar work in the literature (i.e., proposed mechanisms for handling Video Streams of this quality, together with performance evaluation of such mechanisms) prevents us from making result comparisons.

Table 2 and Figure 1 show the results for each movie separately. "The Simpsons" turned out to be the most demanding stream in contrast with "Star Wars" which was the less demanding, although the highest channel throughput appears with the Soccer stream.

Figure 2 and Table 3 show the average results of the simulation runs under all possible movie loads. As the number of video terminals increases, the aggregate bit rate gets smoother, since the superposition of the movies is known from the literature to always be less bursty than a single movie, and the average bandwidth of the superposition is close to the sum of the mean bit rates of the movies. This is proven once more in our study, as the case of the system accommodating only one movie is shown to provide the smallest throughput than any other case of movie load.

The $\lambda$ parameter corresponds to the maximum data message arrival rate (expressed in messages per frame) sustained by the system (i.e. such that the video packet dropping probability $<0.0001$ and the average data message delay $<2$ seconds). 


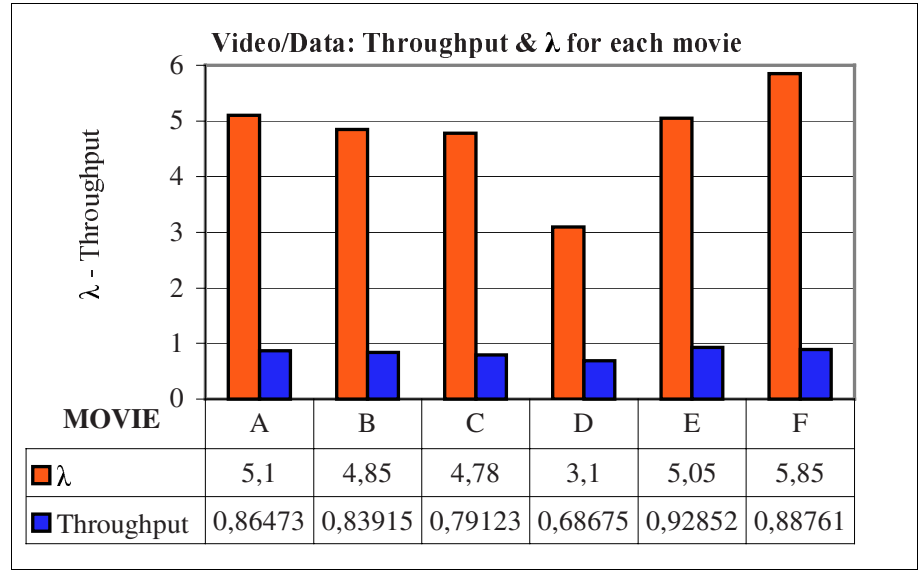

Fig. 1.

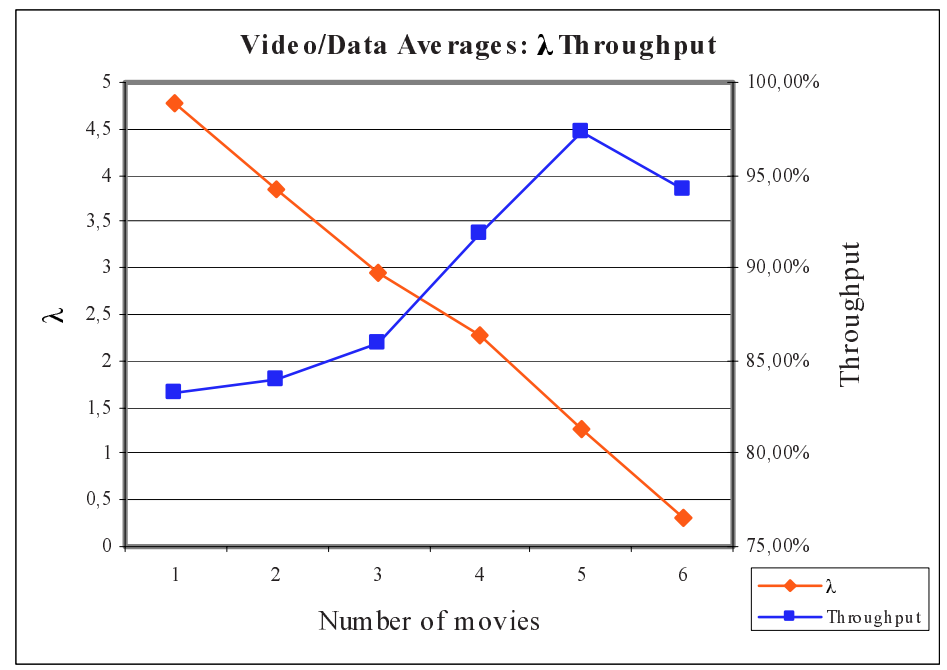

Fig. 2.

Table 3.

\begin{tabular}{|c|c|c|}
\hline System Load & Average $\lambda$ & $\begin{array}{c}\text { Average Throughput } \\
\%\end{array}$ \\
\hline 1 Movie & 4.788333 & 83.29986 \\
\hline 2 Movies & 3.8366 & 83.97877 \\
\hline 3 Movies & 2.96 & 85.90973 \\
\hline 4 Movies & 2.2625 & 91.91385 \\
\hline 5 Movies & 1.266667 & 97.34089 \\
\hline 6 Movies & 0.31 & 94.18723 \\
\hline
\end{tabular}




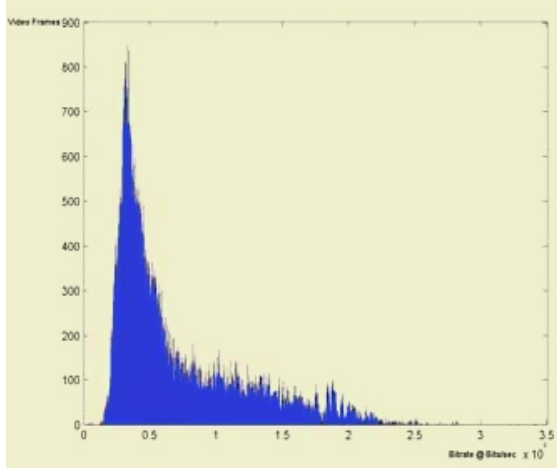

Fig. 3. News Stream

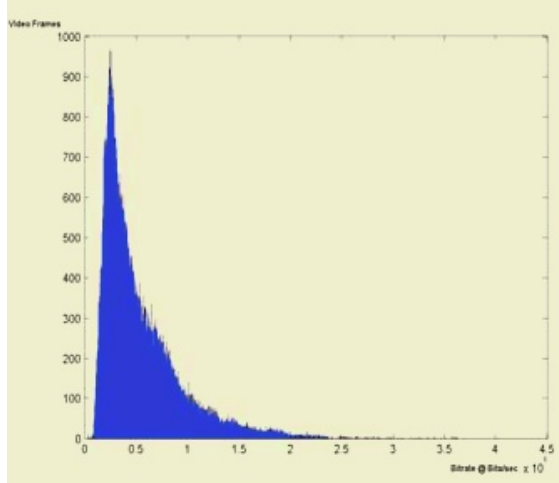

Fig. 5. Silence of the Lambs Stream

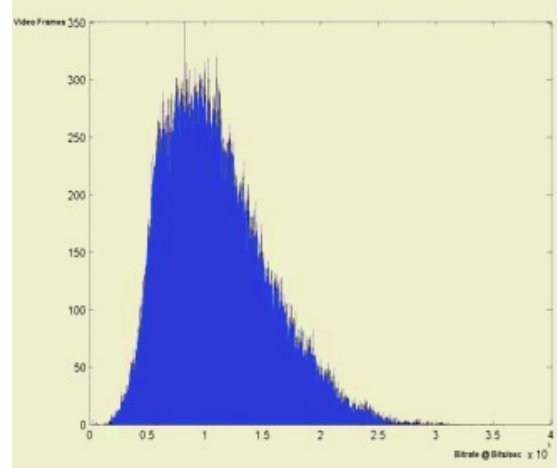

Fig. 7. Soccer Stream

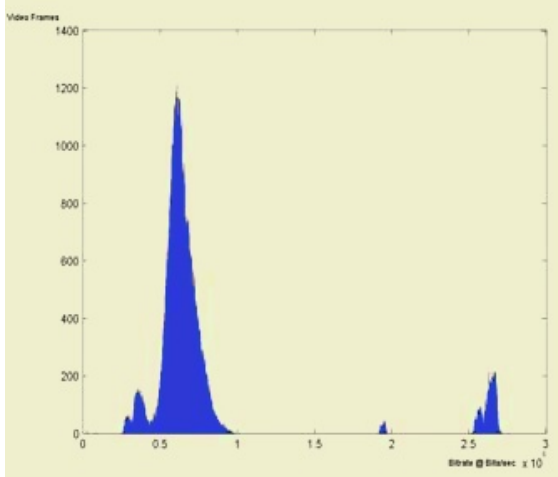

Fig. 4. Parking Lot Camera Stream

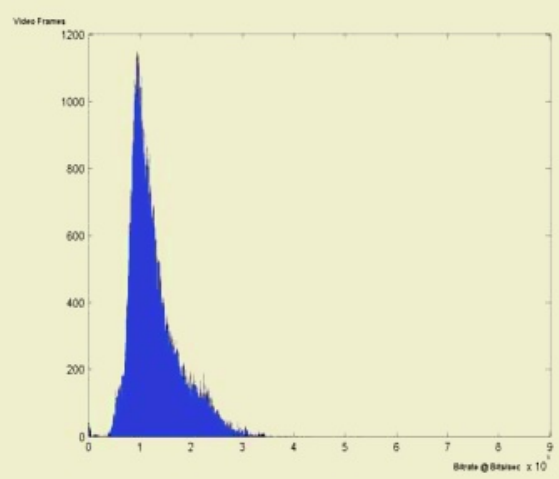

Fig. 6. The Simpsons Stream

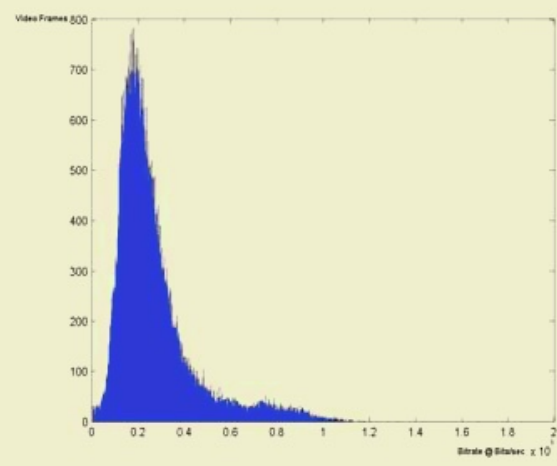

Fig. 8. Star Wars Stream 
Another comment that needs to be made is about the throughput of the system when there are 5 active video terminals. This throughput is remarkably high $(97,3 \%)$, and the reason is that in this case the channel gets filled up with video packets but there is still some empty space for data packets, which almost ideally fill the channel.

On the contrary, when six movies are present in the system, the channel throughput decreases. The reason for this result can be explained if we sum up the peak bit rates of the six movies. The total peak rate reaches $25 \mathrm{Mbps}$, which is significantly higher than the total channel capacity. Of course, the multiplexing gain is also significant and it is rather rare that all of the 6 video terminals would transmit at their peak bit rates at the same time but still their superposition surpasses a few times (i.e., for a few video frames) the channel capacity of $20 \mathrm{Mbps}$. This forces the system to experience, in these video frames, severe video packet dropping, because of the very strict video dropping probability requirements, and therefore leads to the decrease in throughput in comparison with the 5 active video terminals scenario.

A final comment concerns the average data messages delays of our mechanism (the corresponding results are not shown here). In all cases examined in this study, the average data messages delays were found to be roughly constant and approximately equal to 960 msecs ( 80 packets per message * 12 msecs per frame).

This can be explained as follows. In the case of one Video Terminal being active in the system, the maximum data message arrival rate sustained by the system is on average 4.79 messages per frame and the 20 minislots available per frame for resolving the collisions among the contending data requests are enough. Most of the data request packet collisions are resolved within one frame. The corresponding data terminals receive slot reservations in the same frame with high probability, since the utilization of the frame is roughly equal to $83 \%$ and each Data Terminal can be allocated only one reserved slot per frame. Similarly, in the case of six active Video Terminals, the maximum data message arrival rate sustained, is equal to 0.31 messages per frame, and the 20 request minislots are sufficient for handling the new video request packets (at most six in each frame, if all Video terminals undergo a bit rate increase at the same time which happens rarely) and the new data message arrivals, which correspond to one new arrival per three frames on average.

\section{Conclusions}

In this work we proposed a mechanism for transmitting high quality stored multimedia streams and data traffic, over a wireless channel of high capacity. We evaluated its performance through an extensive simulation study in which we used actual MPEG-4 stream traces together with data generated by a synthetic model fitted to measurements of email traffic from a National University and Research Network (FUNET). The results of our study show that the proposed mechanism achieves high aggregate channel throughput in all cases of traffic load, while preserving the Quality of Service (QoS) requirements of each traffic type. 


\section{References}

1. C. Papadimitriou, S. Ramanathan, P. V. Rangan, and S.SampathKumar, "Multimedia information caching for personalized video-on-demand", Comput. Commun., vol. 18, pp. 204-216, Mar. 1995.

2. Y.Guo, S.Sen, and D.Towsley "Prefix Caching assisted Periodic Broadcast: Framework and Techniques to Support Streaming for Popular Videos", in Proceedings of the IEEE ICC 2002 Conference.

3. C. Vassilakis, M. Paterakis and P. Triantafillou: "Video Placement and Configuration of Distributed Video Servers on Cable TV Networks", ACM Multimedia Systems Journal, Vol. 8, No. 2, 2000, pp. 92-104.

4. A. Basso, S. Varakliotis, R. Castagno, "Transport of MPEG-4 over IP/RTP", in Proceedings of the PV 2000 Workshop.

5. D.A. Dyson and Z.J. Haas, "A Dynamic Packet Reservation Multiple Access Scheme for Wireless ATM", ACM / Baltzer MONET Journal, 4(2), pp. 87-89, Jan 1999.

6. Q. Pang, A. Bigloo, et al.,"Service Scheduling for General Packet Radio Service Classes", in Proc. of the IEEE Wireless Communications and Networking Conference 1999 (WCNC '99), New Orleans, USA.

7. P. Koutsakis, S. Psychis and M. Paterakis, "On the Integration of MPEG-4 Video Streams with Voice and E-mail Data Packet Traffic over Wireless Picocellular Networks", in Proceedings of the IEEE PIMRC 2001 Conference, San Diego, USA.

8. P. Koutsakis, M. Paterakis, and S. Psychis, "Integrating Voice, Video and Bursty Data Packet Traffic over Burst-Error Wireless TDMA Channels with Adjustable Request Bandwidth", in Proceedings of the IEEE PIMRC 2001 Conference, San Diego, USA.

9. A.C. Cleary and M.Paterakis, "On the Voice - Data Integration in Third Generation Wireless Access Communication Networks", European Transactions on Telecommunications and Related Technologies, Vol. 5, No. 1, Jan.-Feb. 1994, pp. 11-18.

10. Leonardo Chiariglione, "MPEG-4, why use it?", Telecom Italia Lab - Italy, http://leonardo.telecomitalialab.com/paper/mpeg-4/

11. http://peach.eas.asu.edu/index.html

12. N.Passas D.Skyrianoglou and L.Merakos, "Traffic Scheduling in Wireless ATM Networks", in Proceedings of the IEEE ATM'97 Workshop, Lisbon, Portugal, May 1997.

13. P. Koutsakis, "Medium Access Control and Traffic Policing for Multimedia Traffic Integrated Access in High Capacity Wireless Channels", PhD Dissertation ECE Dept, Technical University of Crete, Chania 2002

14. M. Paterakis and P. Papantoni - Kazakos, "A Simple Window Random Access Algorithm With Advantageous Properties", IEEE Trans. on Inform. Theory, Vol. IT-35, No. 5, September 1989, pp. 1124-1130. 